\title{
Syntheses, Characterization, and Antibacterial Evaluation of $P$. grandiflora Extracts Conjugated with Gold Nanoparticles
}

\author{
A. Murei, ${ }^{1}$ K. Pillay $\mathbb{D D}^{2}{ }^{2}$ and A. Samie $\mathbb{D}^{1}$ \\ ${ }^{1}$ Molecular Parasitology and Opportunistic Infection Research Program, Department of Microbiology, \\ School of Mathematics and Natural Science, University of Venda, Private Bag X5050, Thohoyandou 0950, South Africa \\ ${ }^{2}$ School of Life Sciences, University of Kwa-Zulu-Natal, Private Bag X54001, Durban 4001, South Africa
}

Correspondence should be addressed to A. Samie; samieamidou@yahoo.com

Received 9 July 2021; Revised 10 November 2021; Accepted 11 December 2021; Published 24 December 2021

Academic Editor: Brajesh Kumar

Copyright (c) 2021 A. Murei et al. This is an open access article distributed under the Creative Commons Attribution License, which permits unrestricted use, distribution, and reproduction in any medium, provided the original work is properly cited.

\begin{abstract}
Background. With the recent increase in antibiotic resistance to conventional antibiotics, gold nanoparticles, and medicinal plants, extracts present an interesting alternative. Objectives. This study aimed to synthesize, characterize, and evaluate Pyrenacantha grandiflora Baill extracts and gold nanoparticle conjugates against pathogenic bacteria. Methods. We synthesized gold nanoparticles by chemical and biological methods. The nanoparticles were characterized by the use of UV-visible spectrophotometry, followed by transmission electron microscopy (TEM) and energy-dispersive X-ray analysis (EDX). Gold nanoparticles were conjugated to plant extracts and analyzed with a Fourier-transform infrared spectroscope (FTIR). We determined the antimicrobial activity of the conjugates using well diffusion and the microdilution assays. Results. The UV-visible spectra of gold nanoparticles showed a synthesis peak at $530 \mathrm{~nm}$. FTIR analysis indicated functional biomolecules that were associated with plant extract conjugated gold nanoparticles; the formation of $\mathrm{C}-\mathrm{H}$ group and carbonyl $(\mathrm{C}=\mathrm{O})$ groups, $-\mathrm{OH}$ carbonyl, and $\mathrm{C} \equiv \mathrm{C}$ groups were also observed. Biologically synthesized nanoparticles were star-shaped when observed by TEM with an average size of $11 \mathrm{~nm}$. Gold nanoparticles synthesized with P. grandiflora water extracts showed the largest zone of inhibition (22 mm). When the gold nanoparticles synthesized by the biological method were conjugated with acetone extracts of P. grandiflora, MIC as low as $0.0063 \mathrm{mg} / \mathrm{mL}$ was observed against beta-lactamase producing K. pneumonia. The activity of acetone extracts was improved with chemically synthesized gold nanoparticles particularly when beta-lactamase producing $E$. coli and MRSA were used as test organisms. A synergistic effect was observed against all tested bacteria, except for MRSA when gold nanoparticles were conjugated with acetone extract. Conclusion. Overall, P. grandiflora tuber extracts conjugated with gold nanoparticles showed a very good antibacterial activity that improved both plant extract and gold nanoparticle's individual activity.
\end{abstract}

\section{Introduction}

New strategies are being developed to improve existing medicines to combat antibiotic resistance among pathogenic microorganisms, and the use of inorganic nanoparticulate materials in conjugation with naturally occurring compounds in combination is more and more appreciated [1]. Plant extracts and microorganisms seem to be the best candidates, and they are suitable for large-scale biosynthesis of nanoparticles [2]. Plant-based nanoparticles are more stable, and the rate of synthesis is faster compared to the synthesis by magnetotactic microorganisms. Moreover, the nanoparticles produced from chemical synthesis are more varied in shape and size in comparison with those produced by other organisms [3].

The application of plant-based material in the synthesis of nanoparticles has several advantages over other materials that have attracted the attention of many researchers who have now been very involved in the investigation of the mechanisms of metal ion uptake and reduction by plants. This could be useful in the understanding of the mechanisms leading to the formation of nanoparticles by plant materials [2]. Previous studies have suggested that nanoparticles that are made from noble metals such as silver, platinum, and gold are used worldwide and are contained in products many of which come into direct contact with the human 
body [4]. These products include examples of detergents, shampoos, cosmetic products, as well as toothpaste. This use is beside many other applications including medical and pharmaceutical applications and gold coming first in terms of having a long history of use.

Many civilizations such as those of China and India have used red colloidal gold for medicinal purposes. Similarly, gold nanoparticles have found application in the field of diagnostics as well as for drug delivery [5]. Therefore, there is a growing need to develop environmentally friendly processes for nanoparticle synthesis without using toxic chemicals. In this approach, the surface of the solid core of the metal nanoparticles is functionalized either by noncovalent or covalent interactions [6]. Covalent linking requires additional chemical modification of drug molecules, which could bring about a modification of the properties of drug molecules [7]. Noncovalent strategies to functionalize the drug molecules on the surface of nanoparticles, however, involve only electrostatic and van der Waal's interactions, and accordingly, drug can be utilized in its native form [7]. We have previously reported on the synthesis and evaluation of antibacterial activities of silver nanoparticles using Pyrenacantha grandiflora (also used in the present report) that is a medicinal plant used by local traditional healers for the treatment of gastrointestinal diseases [8]. In the present study, we used gold and P. grandiflora extracts to synthesis nanoparticles and evaluate their antimicrobial activity. This would broaden the scope of the activities covered by this useful plant.

\section{Materials and Methods}

2.1. Chemicals and Reagents Used. Chloroauric acid $\left(\mathrm{HAuCl}_{4}\right)$ was purchased from Sigma (USA). Trisodium citrate was purchased from Rochelle Chemicals (SA). All chemicals were of molecular grade.

2.2. Microorganisms and Growth Media. The microorganisms used in the present study are the same as those used in a previous study by our research group that include Magnetospirillum magnetotacticum MS-1 (DSMZ, Germany). This microorganism was used for the biological synthesis of the gold nanoparticles and was grown in a modified chemically defined growth medium supplemented with Isogro. Methicillin-resistant Staphylococcus aureus (MRSA) ATCC 25923 and methicillin-susceptible Staphylococcus aureus (MSSA) ATCC 33594 were subcultured on mannitol salt agar (Neogen, Michigan), while Escherichia coli ATCC 35218 (beta-lactamase-producing E. coli) and E. coli ATCC 25922 were subcultured on MacConkey agar (Oxoid, England). Klebsiella pneumonia ATCC 700603, which is a beta-lactamase-producing organism, was subcultured on nutrient agar (Rochelle, SA). An inoculum of each bacterial strain (except M. magnetotacticum) was suspended in $5 \mathrm{~mL}$ of Mueller-Hinton broth (Rochelle, SA) and incubated for 3 hours at $37^{\circ} \mathrm{C}$. The cultures were diluted with MuellerHinton broth and adjusted to give a concentration of bacterial cells equivalent to a $0.5 \mathrm{McF}$ arland standard prior to antibacterial testing.

\subsection{Biological Synthesis of Gold Nanoparticles Using Bacteria}

2.3.1. Cultivation of Magnetospirillum magnetotacticum Bacteria. M. magnetotacticum MS-1 was cultured in chemically defined growth media as previously described by Murei and colleagues with slight modification [8]. For gold nanoparticles synthesis, tetrachloroauric acid (Sigma, USA) was added as a source of metal in the growth media. Because MS- 1 cells grow well in microaerobic conditions, they were grown for 4 days at $30^{\circ} \mathrm{C}$ in airtight $50 \mathrm{~mL}$ Falcon tubes wrapped with parafilm. To prevent photodegradation of gold chloride in the media, tubes were covered with foil. The presence of gold nanoparticles in the media was confirmed by a color change from orange to ruby red.

2.3.2. Analysis of Gold Nanoparticles Synthesis by TEM. Magnetospirillum magnetotacticum cells were examined using high-resolution transmission electron microscopy (HR-TEM) to see if they had produced gold nanoparticles. A drop of the bacterial culture was placed onto a carboncoated copper TEM grid to prepare the samples. The sample was then dried for one hour under an infrared lamp. Highresolution TEM images were obtained using a JEOL TEM model no. 2100 instrument set to an accelerating voltage of $200 \mathrm{kV}$ and a resolution of $0.23 \mathrm{~nm}$.

2.3.3. Isolation of Gold Nanoparticles. We used the MACS magnetic separation column to isolate gold nanoparticles from the bacterial culture (Miltenyl Biotec, Germany). In brief, the bacterial cells were suspended in $20 \mathrm{mM}$ HEPES-4 mM EDTA (pH 7.4), and the cell membrane was disrupted by sonication for 5 minutes at $21^{\circ} \mathrm{C}$. Centrifugation at 9,000 rpm for 30 minutes was used to remove the unbroken cells. The supernatant was collected and run through a MACS magnetic separation column according to the manufacturer's instructions. Unbound magnetic particles were washed with $10 \mathrm{mM}$ HEPES-200 mM NaCl (pH 7.4) before being eluted with $10 \mathrm{mM}$ HEPES (pH 7.4). The formation of gold nanoparticles at 500-600 nm was studied using a UV-Vis spectrophotometer.

\subsection{Chemical Synthesis of Gold Nanoparticles}

2.4.1. Gold Nanoparticles Preparation. The gold nanoparticles were prepared according to the method described previously [9]. Polyethylene glycol (PEG) 400 was used as the reducing agent for tetrachloroauric acid. To obtain the PEG-AuNPs, $600 \mu \mathrm{L}$ of PEG400 was dissolved in $95 \mathrm{~mL}$ of water; then $900 \mu \mathrm{L}$ of $1 \%$ sodium hydroxide was added to the PEG solution for $\mathrm{pH}$ adjustment ( $\mathrm{pH}$ around 8). Afterward, $600 \mu \mathrm{L}$ of $2 \% \mathrm{HAuCl}_{4}$ solution was rapidly added to the boiling PEG solution under stirring. The mixture was further boiled for 5 minutes. A wine-red colloid was rapidly formed that confirmed the formation of gold nanoparticles. 
2.4.2. UV-Vis Spectrum Analysis. Characterization of gold nanoparticles was done using UV-visible spectrophotometry (Specord 210, Analytikjena spectrometer). The reduction of chloroauric acid to gold nanoparticles by PEG was confirmed by observing a broad absorbance peak between 500 and $600 \mathrm{~nm}$.

\subsubsection{High-Resolution Transmission Electron Microscopy.} Further characterization was done using HR-TEM studies. The sample was prepared by placing a drop of the nanoparticle solution onto a carbon-coated copper TEM grid. The sample was then dried under an infrared lamp for a period of $45 \mathrm{~min}$ for the solvent to evaporate. High-resolution TEM images were obtained on a JEOL TEM model no. 2100 instrument, operated at an accelerating voltage of $200 \mathrm{kV}$ and $0.23 \mathrm{~nm}$ resolution.

\subsection{Preparation of Plant Extracts Conjugated with Gold} Nanoparticles. Acetone, methanol, and water were used as solvents to prepare $P$. grandiflora extracts as described by Murei and colleagues [8]. In brief, the bioactive compounds of $P$. grandiflora tuber were extracted as follows: for the hot water extract, the stock solution was prepared by combining $100 \mathrm{~g}$ of tuber powder in $1 \mathrm{~L}$ of distilled water, boiling for 15 minutes, and then cooling and storing at $4^{\circ} \mathrm{C}$. In order to extract methanol and acetone, $100 \mathrm{~g}$ of dried tuber were mixed with $1 \mathrm{~L}$ of each solvent and allowed to homogenize for 24 hours at room temperature. All of the mixtures were filtered using Whatman filter paper. Rotary evaporators (Rotavapor-R, Buchi, Switzerland) were used to concentrate filtrates at $50^{\circ} \mathrm{C}$ for acetone and $60^{\circ} \mathrm{C}$ for methanol. The freeze dryer was used to concentrate hot water extract. At room temperature, all concentrated samples were dried into a powder. A Fourier-transform infrared spectrophotometer (FTIR) was used to detect different functional groups responsible for biological activities in the P. grandiflora tubers crude extracts.

2.6. Gold Nanoparticles and P. grandiflora Extract Conjugate Preparation. Noncovalent modification of plant extract with gold nanoparticles was applied in the generation of hybrid molecules. Synthesized gold nanoparticles from both chemical and biological methods were mixed with $2 \mathrm{~mL}$ of $10 \mathrm{mg} / \mathrm{mL}$ of each plant extract (acetone, water, and methanol extracts) and incubated at $4^{\circ} \mathrm{C}$ for 24 hours.

2.7. Characterization of Conjugates. To detect various functional groups formed after conjugation that may be responsible for biological activities, all conjugates were analyzed using a Fourier-transform infrared spectrophotometer (FTIR) in the $400-4,000 \mathrm{~cm}^{-1}$ range. Approximately $500 \mu \mathrm{L}$ of dried conjugates were placed in the sample chamber of an FTIR spectrophotometer, and spectra were recorded in the scan range of $400-4,000 \mathrm{~cm}^{-1}$ with a resolution of $4 \mathrm{~cm}^{-1}$ on a Nicolet Avatar 330 FTIR spectrophotometer.

\subsection{Antimicrobial Activities of AuNPs and P. grandiflora Extracts and Conjugates}

2.8.1. Well Diffusion Assay. We determined the antimicrobial activities of the nanoparticles conjugates by the use of the well diffusion method. The inhibition zones were measured in millimeters $(\mathrm{mm})$. Briefly, bacterial suspensions with a turbidity of $0.5 \mathrm{McF}$ arland were prepared for the test. Beta-lactamase-producing E. coli and K. pneumonia, as well as the $S$. aureus isolates were inoculated onto MuellerHinton agar plates. Using a cork borer, wells with a diameter of $6 \mathrm{~mm}$ were cut and filled with $30 \mu \mathrm{L}$ of the conjugates and reference samples (plant extracts and gold nanoparticles). As a negative control, distilled water was used. Plates were incubated at $37^{\circ} \mathrm{C}$ for 24 hours. The diameters of the growth inhibition zones were measured after incubation.

2.8.2. Microdilution Assay. The minimum inhibitory concentration (MIC) was defined as the lowest dilution of all extracts plus conjugate samples that inhibited microbial growth [10]. The negative control was distilled water, and the positive control was gentamycin. The results were read after adding INT (iodonitrotetrazolium) by observing the color change and determining the MIC. The extracts and AuNPs that demonstrated activity (no color change) were reinoculated in a sterile agar plate and incubated overnight to determine the minimum bactericidal concentration.

2.8.3. Fractional Inhibition Concentration Index (FICI) Calculations. The fractional inhibition concentration index (FICI) was calculated and used to evaluate the mutual influence of $P$. grandiflora tuber extracts and gold nanoparticles in the conjugate by the following formula:

$$
\text { FICI }=\frac{\text { MIC of } A B}{\text { MIC of } A}+\frac{\text { MIC of } A B}{\text { MIC of } B},
$$

where $A B$ represents a combination of $P$. grandiflora tuber extracts $(A)$ and gold nanoparticles $(B)$. Results were interpreted as synergy $(\mathrm{FICI} \leq 0.5)$, antagonism $(\mathrm{FICI}>4)$, or no interaction, or additive (FICI $>0.5-4.0$ ).

\section{Results}

3.1. TEM Analysis of Biologically Synthesized Gold Nanoparticles. After incubation with gold, the color of the media containing the bacteria changed to indicate the formation of the gold nanoparticles. The cell morphology of the bacteria used for the production of the nanoparticles was evaluated by HR-TEM (Figure 1(a)) that also revealed the presence of the gold nanoparticles within the cells (Figure 1(b)). Synthesized gold nanoparticles were starshaped with their sizes ranging from 9 to $30 \mathrm{~nm}$. The selected diffraction patterns (Figure 1(c)) indicated that these gold nanoparticles were formed from the reduction of metal ions. The EDX analysis confirmed that the particles were composed of elemental gold (Figure 1(d)). 


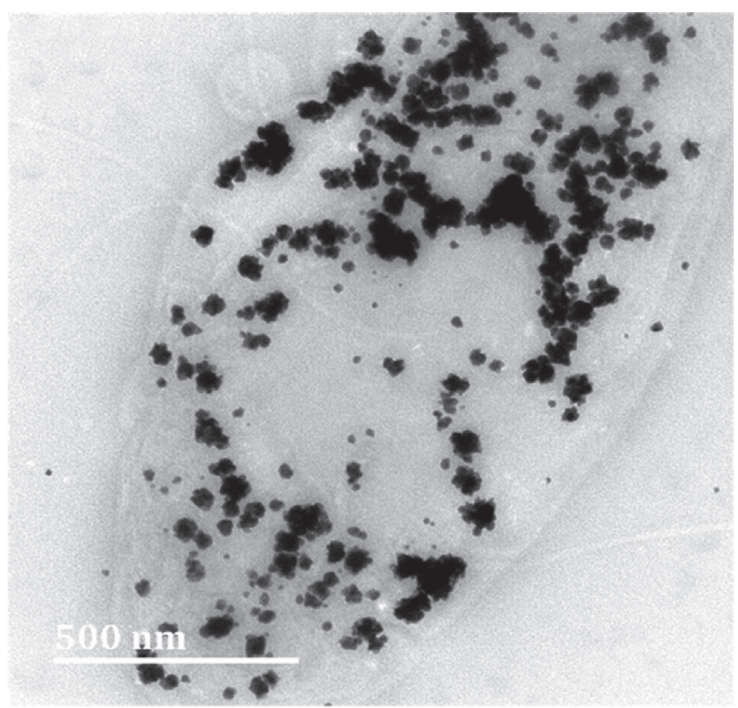

(a)

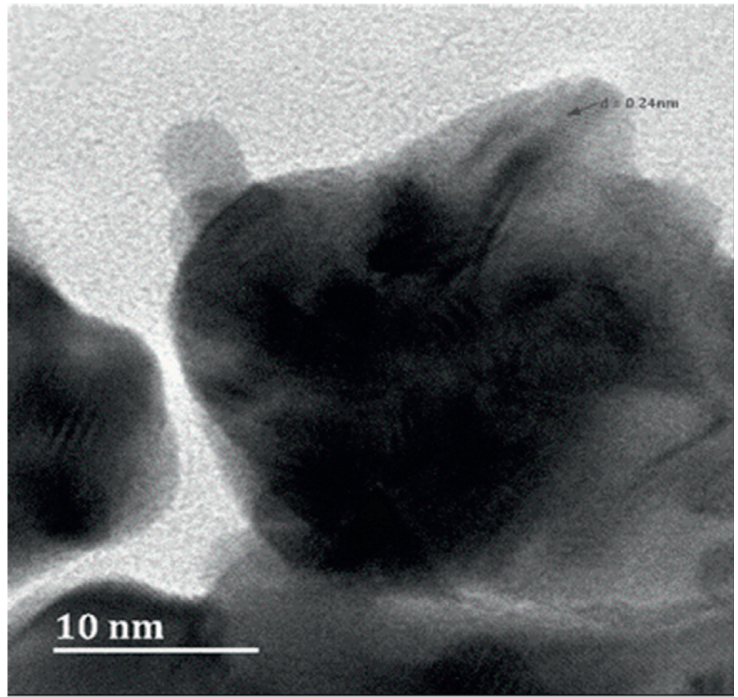

(c)

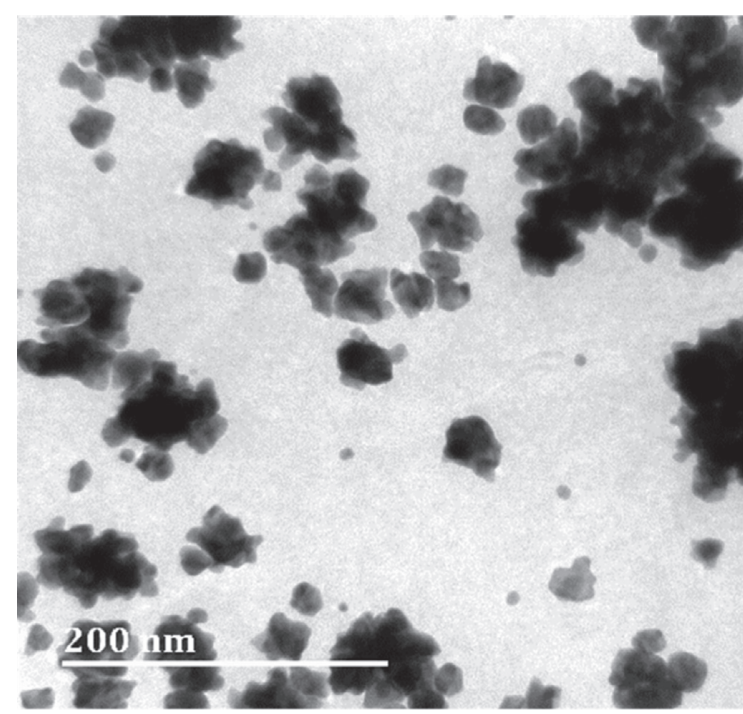

(b)

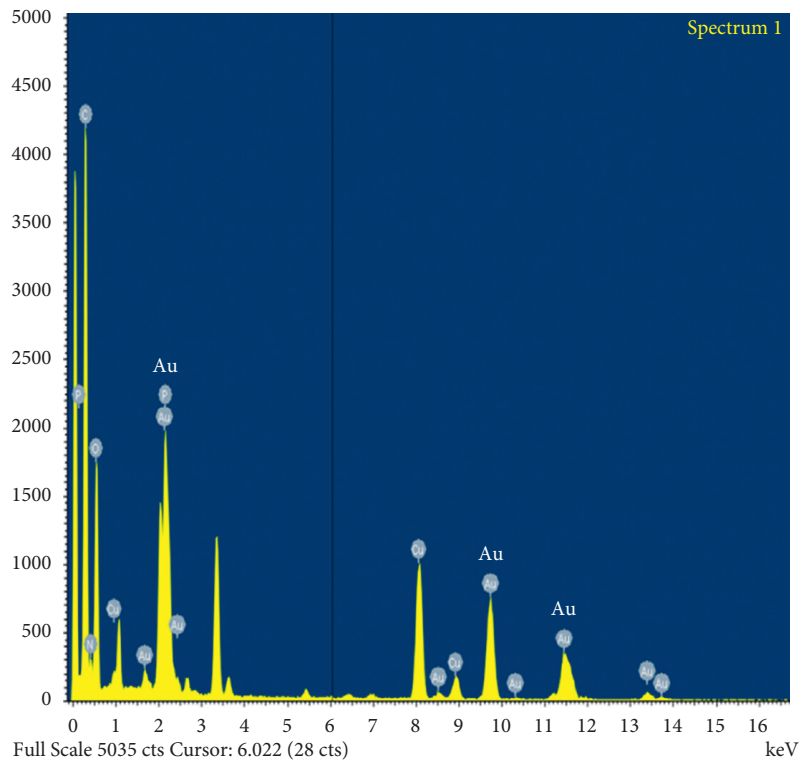

(d)

FIGURE 1: (a) TEM images of Magnetospirillum magnetotacticum with gold nanoparticles, (b) isolated gold nanoparticles, (c) lattice fringes, and (d) EDX pattern.

3.2. Analysis of Chemically Synthesized (PEG Capped) Gold Nanoparticles. Gold nanoparticles capped with PEG were successfully synthesized using the chemical method. Synthesis of gold nanoparticles was initially confirmed by a color change in PEG-gold chloride solution from pale yellow (Figure 2(a)) to a ruby red color (Figure 2(b)), giving rise to a characteristic absorption of light at a wavelength of $500-600 \mathrm{~nm}$.

3.3. UV-Visible Analysis of Chemically Synthesized Gold Nanoparticles. UV-visible spectroscopic studies of chemically synthesized gold nanoparticles showed the peak at $530 \mathrm{~nm}$ (maximum absorbance) that confirms the presence of gold nanoparticles as shown in Figure 3.
3.4. TEM Analysis of Chemically Synthesized Gold Nanoparticles. TEM revealed that the morphology of nanoparticles is spherical in shape. In the higher magnification image, gold nanoparticles of $7-16 \mathrm{~nm}$ diameter were also observed. However, the average size of the nanoparticles was found to be $11 \mathrm{~nm}$. Figure 4(a) shows the TEM picture of gold nanoparticles. The elemental composition was confirmed by EDX (Figure 4(b)).

3.5. Analysis of FTIR Spectra of P. grandiflora Extracts. The FTIR spectra of $P$. grandiflora extracts revealed different characteristic peak values for numerous functional compounds. The extracts revealed the presence of hydroxyl groups $(\mathrm{OH}), \mathrm{C}-\mathrm{H}$ stretching, and $\mathrm{C}=\mathrm{C}$ carboxyl groups 


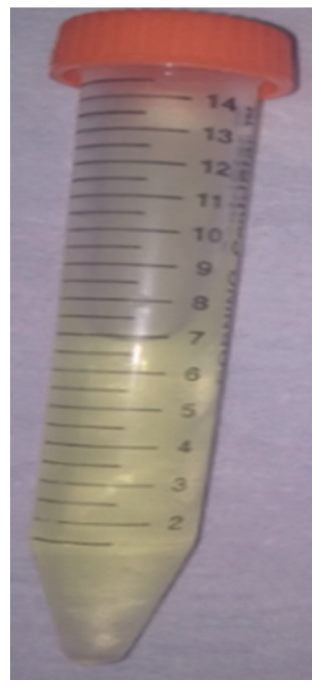

(a)

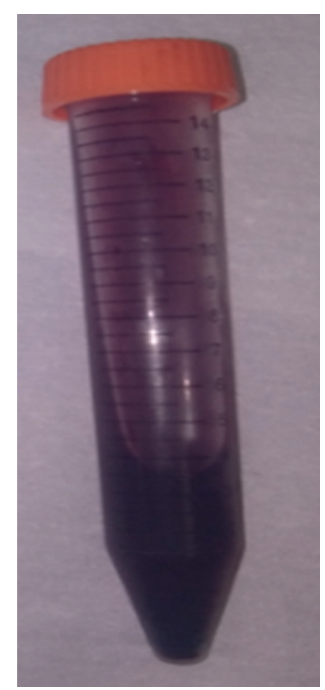

(b)

FIGURE 2: Images illustrating the color change of PEG-gold chloride solution from pale yellow (a) to ruby red (b) to confirm the synthesis of gold nanoparticles.

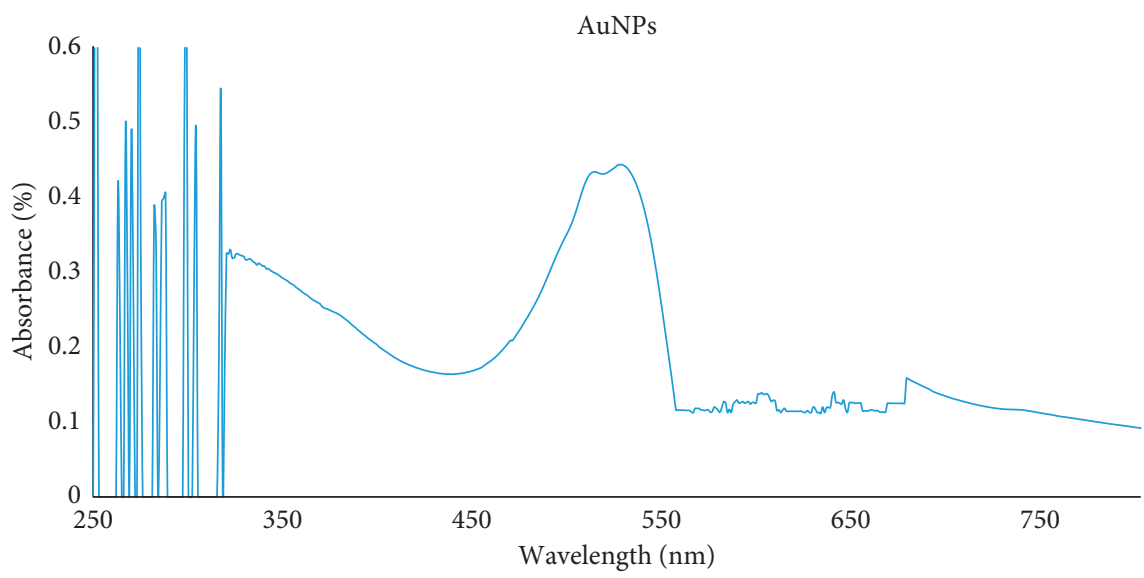

FIGURE 3: UV-visible spectrum of chemically synthesized gold nanoparticles.

ranging from 3306-3153, 2923-2894, and 1689-1557, respectively. Hence, these functional groups observed in different $P$. grandiflora extracts probably indicate the presence of various phytochemicals and other medicinal important compounds.

3.6. Analysis of Gold Nanoparticles Conjugated with Plant Extracts. Six conjugates were made from gold nanoparticles and plant extracts, three from chemically synthesized gold nanoparticles, and three from biologically synthesized gold nanoparticles. Conjugates were gold nanoparticles-methanol extract $(\mathrm{GM})$, gold nanoparticles-water extract $(\mathrm{GW})$, and gold nanoparticles-acetone extract (GA). The measurements of FTIR were carried out in order to recognize the existence of different functional groups that resulted after conjugation (Figure 5). Most conjugated samples compared to reference samples showed the formation of $\mathrm{C}-\mathrm{H}$ group and carbonyl $(\mathrm{C}=\mathrm{O})$ group; however, $-\mathrm{OH}$ carbonyl was also observed.

3.7. Well Diffusion Assay. The antibacterial activities of $P$. grandiflora tuber extracts conjugated with gold nanoparticles were evaluated using well diffusion assay. Five pathogenic bacterial ATCC strains were used to evaluate the efficacy of the conjugates. Unconjugated biologically synthesized nanoparticles showed the smallest zone of growth inhibition of $6 \mathrm{~mm}$ in diameter in all bacteria tested in this study except in E. coli ATCC 35218 that was $7 \mathrm{~mm}$ in diameter (Figure 6). However, the highest zone of growth inhibition of $22 \mathrm{~mm}$ was observed when conjugated with water extract against methicillin-susceptible Staphylococcus aureus and gold nanoparticles conjugated with acetone extract against methicillin-susceptible Staphylococcus aureus. 


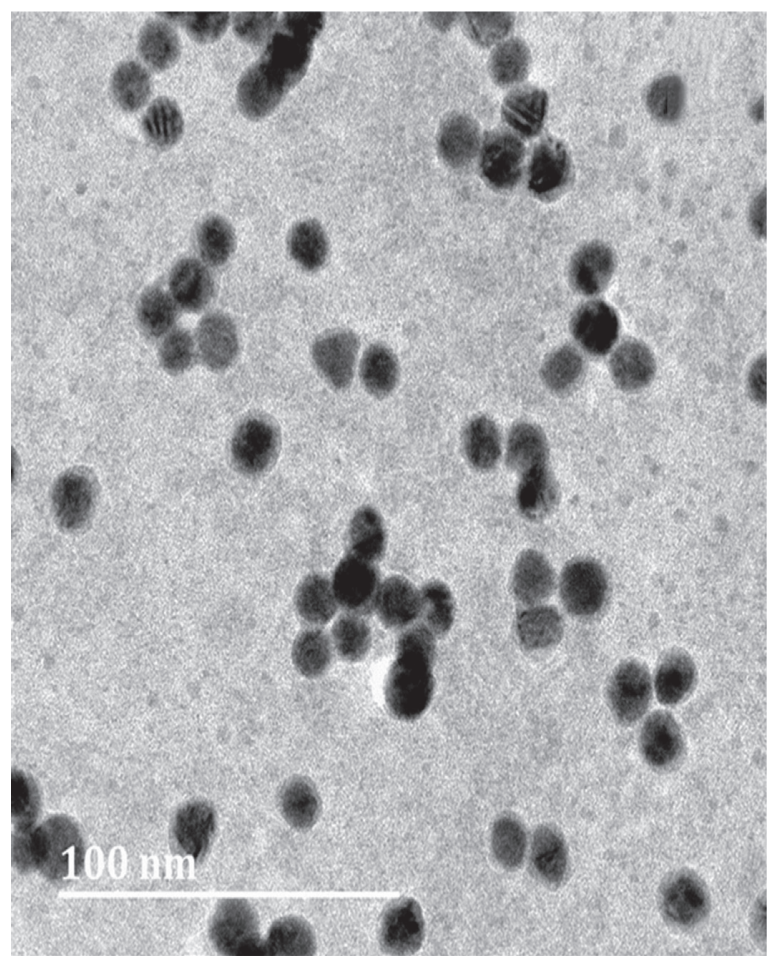

(a)

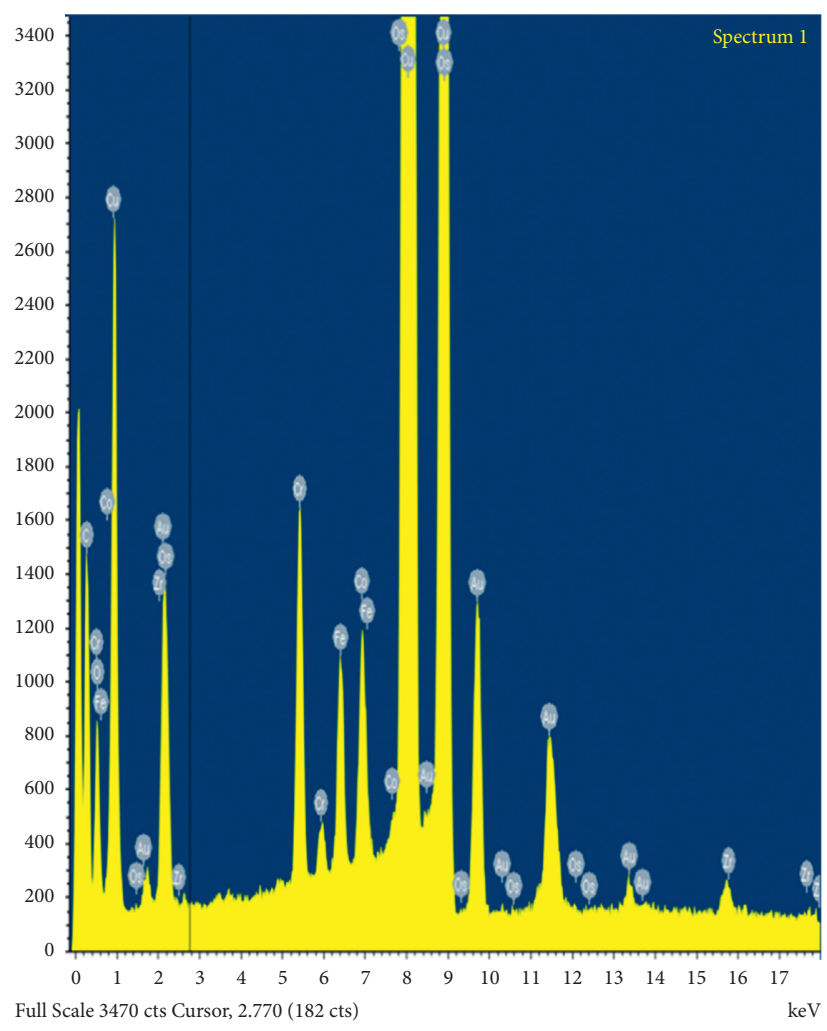

(b)

FIGURE 4: (a) TEM images of chemically synthesized gold nanoparticles and (b) EDX patterns.

Chemically synthesized gold nanoparticles and their conjugates exhibited good antibacterial activity against all tested bacterial strains with a diameter ranging from 14 to $27 \mathrm{~mm}$ (Figure 7). The highest antibacterial activity $(27 \mathrm{~mm})$ was exhibited by gold nanoparticles alone against MSSA. Klebsiella pneumoniae showed the smallest zone of inhibition of all chemically synthesized gold nanoparticles and conjugates with the lowest zone of growth inhibition diameter of $14 \mathrm{~mm}$ when compared to other bacterial strains. However, overall good antibacterial activity was observed against E. coli ATCC 35218 with a range of $22-25 \mathrm{~mm}$ of growth inhibition zone.

3.8. Microdilution Assay. Microdilution assay was used to determine the minimum inhibitory concentration of $P$. grandiflora tuber extracts conjugated with biologically and chemically synthesized gold nanoparticles. The concentration used ranged from 0.8 to $0.0063 \mathrm{mg} / \mathrm{mL}$ of plant extract conjugated gold nanoparticles. All plant extracts showed good antibacterial activity when conjugated with gold nanoparticles (Table 1). The lowest MIC value of $0.0063 \mathrm{mg} / \mathrm{mL}$ was observed when biologically synthesized gold nanoparticles are conjugated with acetone and water extract against $K$. pneumonia. Chemically synthesized gold nanoparticles also showed the lowest MIC value of $0.0063 \mathrm{mg} / \mathrm{mL}$ against E. coli 25922 and MSSA when conjugated with acetone extract.
3.8.1. Minimum Bactericidal Concentration (MBC). Only unconjugated biologically synthesized gold nanoparticles and their conjugate with acetone extract were able to kill E. coli 25922 with $\mathrm{MBC}$ values of 0.05 and $0.8 \mathrm{mg} / \mathrm{mL}$. None of the chemically synthesized gold nanoparticles conjugates were able to exhibit bactericidal activity.

3.9. Fractional Inhibition Concentration Index (FICI) Calculations. Fractional inhibition concentration index was calculated based on the results obtained from MIC, and the results were recorded in Table 2. A total of six samples from biologically and chemically synthesized gold nanoparticles conjugated plant extracts were tested against five bacterial ATCC strains. A total of 8 synergies (26.7\%) were observed, $12(40 \%)$ were additive, and $10(33.3 \%)$ were antagonism.

\section{Discussion}

Most of the nanoparticles are modified with functionalizing agents in order to conjugate various drugs for successful application for therapeutic purposes [4]. In this study, gold nanoparticles were conjugated with $P$. grandiflora tuber extracts, and their antibacterial activities were evaluated. Gold nanoparticles were synthesized using biological (produced by a bacterium) and chemical methods. The biological synthesis of gold nanoparticles with Magnetospirillum magnetotacticum was chosen due to several advantages such as being a simple, single-step, 

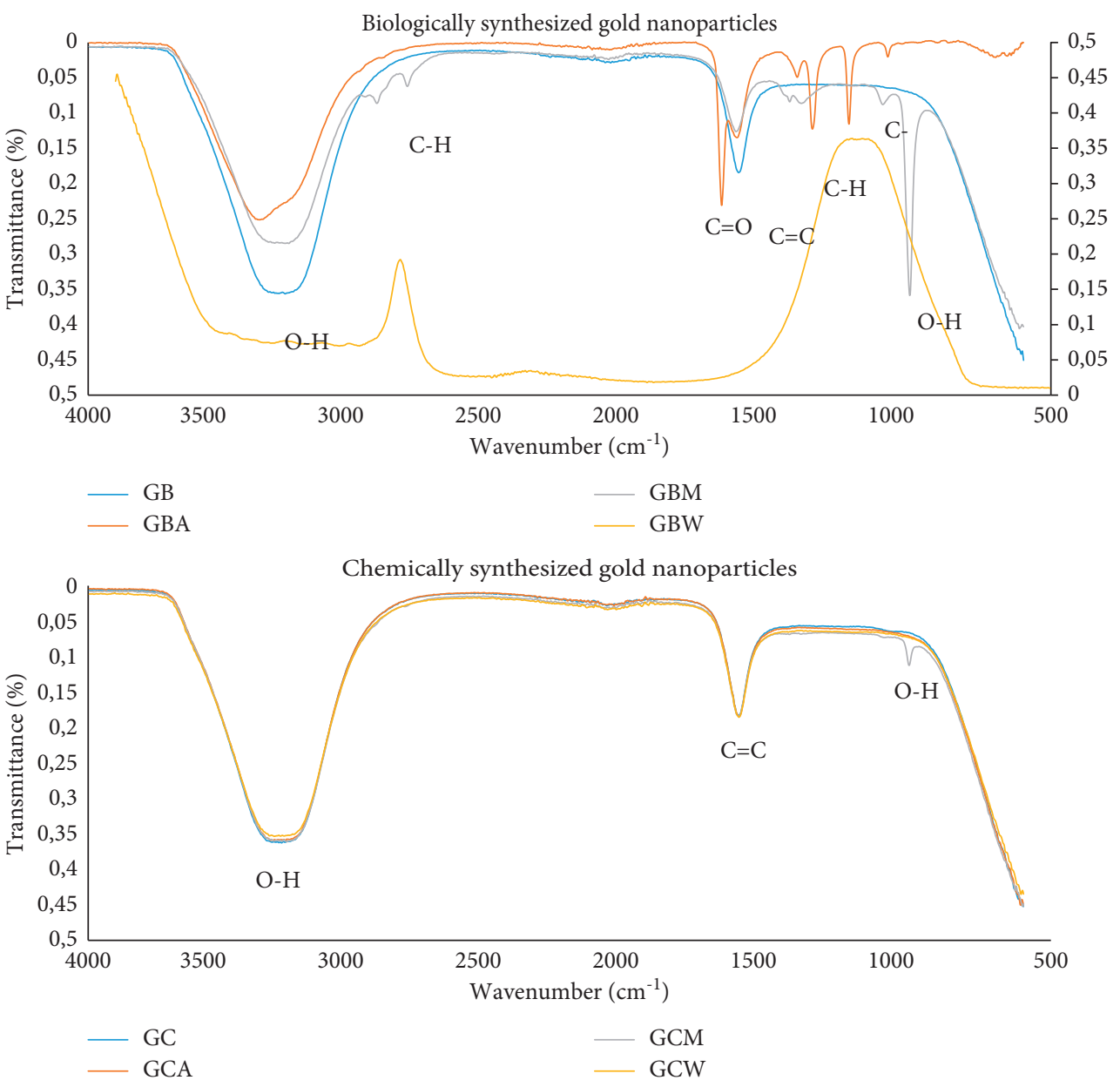

FIGURE 5: FTIR spectra of plant extracts conjugated with gold nanoparticles. GB: biologically synthesized gold nanoparticles, GBA: biologically synthesized gold nanoparticles and acetone extract, GBM: biologically synthesized gold nanoparticles and methanol extract, GBW: biologically synthesized gold nanoparticles and water extract, GC: chemically synthesized gold nanoparticles, GCA: chemically synthesized gold nanoparticles and acetone extract, GCM: chemically synthesized gold nanoparticles and methanol extract, and GCW: chemically synthesized gold nanoparticles and water extract.

environmentally friendly, cost-effective process and due to the biocompatible nature of the synthesized gold nanoparticles [11]. Additionally, previous studies have suggested that there is no need to add any external stabilizing agents because the biogenic components of these bacteria act as stabilizing as well as capping agents [12]. Studies by different authors have shown that substances produced by both plants and microorganisms, such as vitamins, organic acids, amino acids, proteins, as well as idiolites including heterocyclic compounds, polyphenols, alkaloids, and flavonoids, play important role in the reduction of metal salt. Furthermore, these very same metabolites play a role as capping and stabilizing agents during the synthesis of nanoparticles [13]. However, unlike in the biological synthesis of nanoparticles, stabilizing agents are needed in chemically synthesized gold nanoparticles. Therefore, some studies have used polyethylene glycol (PEG) that an example of the hydrophilic segment containing biodegradable copolymers, for surface coating of gold nanoparticles. Studies showed that PEG conformation at the nanoparticle surface is of utmost importance for the opsonin repelling function of the PEG layer
[14]. Further studies will have to determine the stability of the synthesized nanoparticles with or without the stabilizing agents as well as their cytotoxicity in order to gauge the real impact of such substances as good antimicrobials.

Most microorganism-based syntheses of nanoparticles are slow with low productivity. To overcome such hindrances, the growth of the microorganisms was enhanced by the addition of Isogro to the media, and this also increased the yield of the synthesized nanoparticles. Furthermore, issues associated with the production of nanoparticles by microorganisms have been complicated by the maintenance and growth conditions of these organisms. This makes the development of more effective media a research priority. Several organisms have been reported to produce gold nanoparticles in an extracellular fashion, and these include fungal organisms such as Fusarium oxysporum as well as actinomycetes such as Themomonospora spp., [15]. In this study, Magnetospirillum magnetotacticum has synthesized gold nanoparticles intracellularly. Several studies reported the intracellular synthesis of gold nanoparticles by Magnetospirillum sp. as well [12]. 


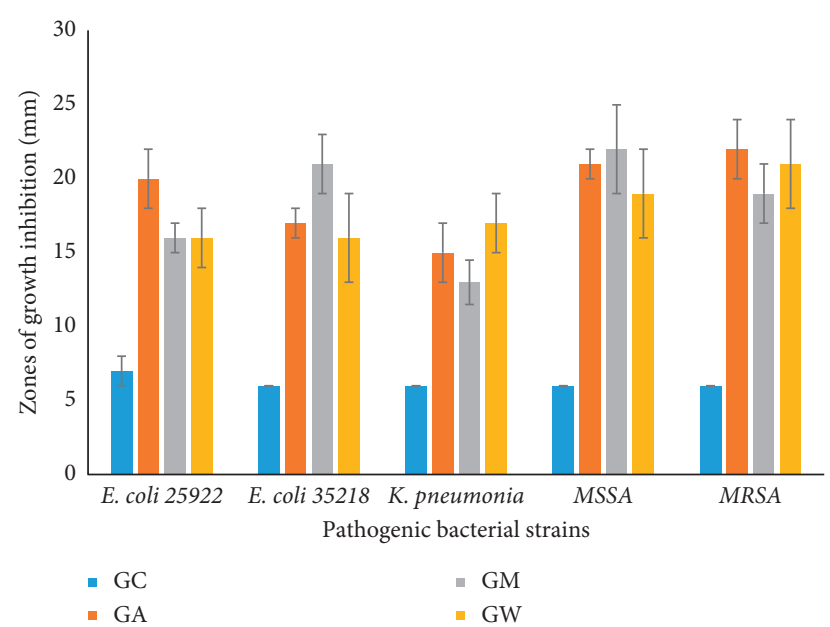

Figure 6: Antibacterial activity of $P$. grandiflora tuber extract conjugates with biologically synthesized gold nanoparticles against selected pathogenic bacteria. GB: biologically synthesized gold nanoparticles, GA: gold nanoparticles and acetone extract, GM: gold nanoparticles and methanol extract, and GW: gold nanoparticles and water extract.

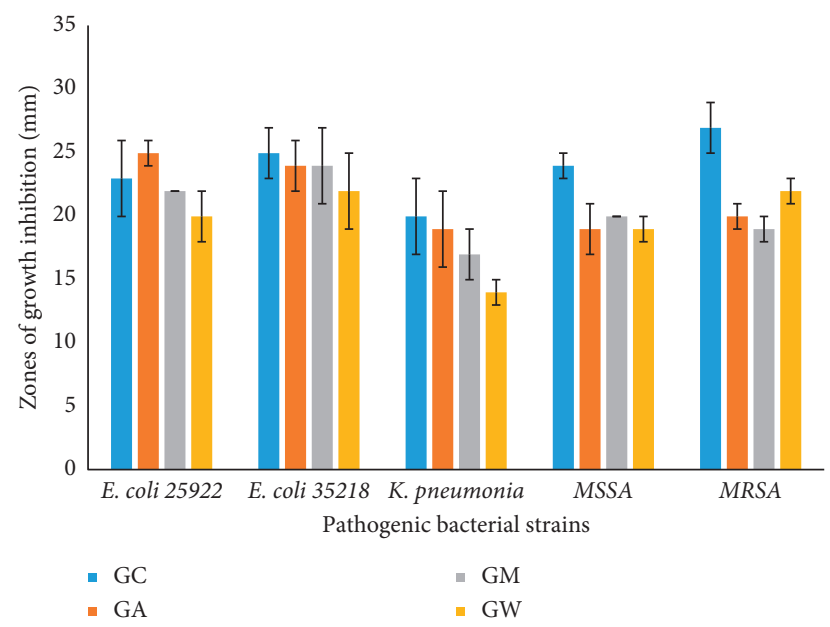

FIGURE 7: Antibacterial activity of $P$. grandiflora tuber extract conjugated with chemically synthesized gold nanoparticles against selected pathogenic bacteria. GC: chemically synthesized gold nanoparticles, GA: gold nanoparticles and acetone extract, GM: old nanoparticles and methanol extract, and GW: gold nanoparticles and water extract.

Southam and Beveridge [16] have demonstrated that gold particles of nanoscale dimensions may readily be precipitated within bacterial cells by incubation of the cells with $\mathrm{Au}^{3+}$ ions. AuNPs of $13 \mathrm{~nm}$ and above are considered noncytotoxic [17]. In this study, TEM revealed the synthesis of 7-16 nm with the chemical method and 9-30 nm nanoparticles with the biological method, which further supports the size of AuNP obtained from TEM [18, 19]. Nanoparticles below $2 \mathrm{~nm}$ have been shown to possess active cytotoxic characteristics [20]. Lengke et al. [21] claimed the synthesis of gold nanostructures in different shapes (spherical, cubic, and octahedral) by filamentous cyanobacteria from $\mathrm{Au}$ (III) chloride complexes. Therefore, the controlled size and shape of gold nanoparticles in the future will be interesting. The EDX pattern obtained is consistent with earlier reports [22].

Conjugations were identified by observing new functional groups with FTIR spectroscopy. After the conjugation process, the formation of $\mathrm{C}-\mathrm{O}$ groups and $\mathrm{OH}$ hydroxyl group on conjugated plant extracts showed the presence of the new bands at 1,536 and $1,095 \mathrm{~cm}^{-1}$. These results indicated that the conjugation between the gold nanoparticles and plant extracts actually occurred. Surface-bound proteins have been reported to stabilize gold nanoparticles through cysteine residues as well as free amino groups [23]. Other studies have reported on the ability of plant extracts to confer liquid-like characteristics to nanoparticles such as those of Cymbopogon flexuosus and the gold nanoparticles with spherical shape by the ketones contained in the plant extracts playing a major role in this context [24].

The mechanisms of antimicrobial activity of nanoparticles are still being studied. However, it has been proposed that gold nanoparticles act on microorganisms through a cell wall. For Gram-positive bacteria, the peptidoglycan layer that is thick and is part of the cell wall makes it difficult for the nanoparticles to penetrate these organisms, while the cell wall of Gram-negative bacteria is thinner and therefore might be more susceptible to the nanoparticles [25]. Minimum inhibition concentration of $P$. grandiflora tuber extracts conjugated with biologically and chemically synthesized gold nanoparticles was determined using a microdilution assay. The concentration used ranged from 0.8 to $0.0063 \mathrm{mg} / \mathrm{mL}$, even though it has been reported that some plant extracts alone do not work [26], all plant extracts show very good antibacterial activity when conjugated with gold nanoparticles. In 2018, Mostafa and colleagues revealed that it is not easy for plant extracts to completely exhibit a bactericidal effect [27]. Yet only a few chemical synthesized gold nanoparticles and their conjugate to acetone extracts were able to kill E. coli 25922. The gold nanoparticles synthesized biologically did not have bactericidal activity since none of the conjugates exhibited MBC. Therefore, chemically synthesized gold nanoparticles are good for bactericidal activity compared to biologically synthesized gold nanoparticles. The present study demonstrated strong antimicrobial activities by the tubers extracts with MIC as low as $0.06 \mathrm{mg} / \mathrm{ml}$ [28].

Increasing the activity of compounds against microorganisms is one of the main objectives of developing complexes such as nanoparticles conjugated to medicinal plants or even some antibiotics. The synergistic effects of gold nanoparticles conjugated with plant extracts were investigated against selected pathogenic bacteria using microdilution assay, and the effects were evaluated by the determination of the fractional inhibition concentration index (FICI). A total of 8 synergies (26.7\%), 12 additive (40\%), and 10 antagonisms (33.3\%) were observed for both biologically and chemically synthesized gold nanoparticles conjugated $P$. grandiflora tuber extracts. In the previous study, we have found increased activity (synergy) when we functionalized commonly used antibiotics and silver nanoparticles [29]. Similarly, other authors have described 
TABLE 1: Minimum inhibitory concentration (MIC) of biologically and chemically synthesized gold nanoparticles as well as their conjugates with P. grandiflora extracts.

\begin{tabular}{|c|c|c|c|c|c|}
\hline Samples & E. coli (ATCC 25922) & E. coli (ATCC 35218) & K. pneumonia (ATCC 700603) & MSSA & MRSA \\
\hline $\mathrm{G}_{\mathrm{B}}$ & 0.05 & 0.1 & 0.0125 & 0.05 & 0.8 \\
\hline $\mathrm{GA}_{\mathrm{B}}$ & 0.05 & 0.1 & 0.0063 & 0.2 & 0.2 \\
\hline $\mathrm{GM}_{\mathrm{B}}$ & 0.4 & 0.2 & 0.8 & 0.05 & 0.2 \\
\hline $\mathrm{GW}_{\mathrm{B}}$ & 0.4 & 0.8 & 0.0063 & 0.1 & 0.2 \\
\hline $\mathrm{G}_{\mathrm{C}}$ & 0.4 & 0.8 & 0.8 & 0.025 & 0.1 \\
\hline $\mathrm{GA}_{\mathrm{C}}$ & 0.0063 & 0.1 & 0.1 & 0.0063 & 0.8 \\
\hline $\mathrm{GM}_{\mathrm{C}}$ & 0.05 & 0.1 & 0.1 & 0.05 & 0.4 \\
\hline $\mathrm{GW}_{\mathrm{C}}$ & 0.1 & 0.1 & 0.1 & 0.05 & 0.1 \\
\hline
\end{tabular}

B: biological synthesis, C: chemical synthesis, G: gold nanoparticles, GA: gold nanoparticles with acetone extracts, GM: gold nanoparticles with methanol extracts, and GW: gold nanoparticles with water extracts.

TABLe 2: Effect of conjugating P. grandiflora tuber extracts with gold nanoparticles.

\begin{tabular}{|c|c|c|c|c|c|}
\hline Samples & E. coli (ATCC 35218) & E. coli (ATCC 35218) & K. pneumonia (ATCC 35218) & MSSA & MRSA \\
\hline $\mathrm{GA}_{\mathrm{B}}$ & $1.125(\mathrm{~A})$ & $1.125(\mathrm{~A})$ & $0.511875(S)$ & $4.25(\mathrm{~N})$ & $0.75(\mathrm{~A})$ \\
\hline $\mathrm{GM}_{\mathrm{B}}$ & $16(\mathrm{~N})$ & $3(\mathrm{~A})$ & $66(\mathrm{~N})$ & $1.080645(\mathrm{~A})$ & $2.25(\mathrm{~A})$ \\
\hline $\mathrm{GW}_{\mathrm{B}}$ & $16(\mathrm{~N})$ & $10(\mathrm{~N})$ & $1.504(\mathrm{~A})$ & $17.87302(\mathrm{~N})$ & $0.5(S)$ \\
\hline $\mathrm{GA}_{\mathrm{C}}$ & $0.0315(S)$ & $0.25(S)$ & $0.25(S)$ & $0.259875(\mathrm{~S})$ & $10(\mathrm{~N})$ \\
\hline $\mathrm{GM}_{\mathrm{C}}$ & $1.125(\mathrm{~A})$ & $0.625(\mathrm{~A})$ & $0.375(S)$ & $2.080645(\mathrm{~A})$ & $8(\mathrm{~N})$ \\
\hline $\mathrm{GW}_{\mathrm{C}}$ & $2.25(\mathrm{~A})$ & $0.375(\mathrm{~S})$ & $15.99802(\mathrm{~N})$ & $9.936508(\mathrm{~N})$ & $1.125(\mathrm{~A})$ \\
\hline
\end{tabular}

B: biological synthesis, C: chemical synthesis, A: additive, N: antagonism, S: synergy, G: gold nanoparticles, GA: gold nanoparticles with acetone extracts, GM: gold nanoparticles with methanol extracts, and GW: gold nanoparticles with water extracts. Bold values show synergistic results after the combination has been used. This can be seen by the addition of $S$ indicating synergy.

the increase of the antimicrobial activity of conjugated nanoparticles suggesting that such combinations are beneficial in the fight against infectious diseases. For example, in 2007, Rosato et al. reported synergistic interactions of gold nanoparticles conjugated with norfloxacin against $S$. aureus, Streptococcus mutans, E. coli ATCC 25922, and P. aeruginosa [30]. These synergistic activities of conjugates suggest that it might be possible to reduce the viability of bacterial strains at lower antibiotic concentrations.

\section{Conclusion}

This study successfully synthesized gold nanoparticles using chemical and biological methods and conjugated them with $P$. grandiflora extracts. The chemically synthesized nanoparticles were very effective and had a very small size that makes them ideal for drug delivery applications. Biologically synthesized gold nanoparticles and their conjugates with acetone appeared to be bactericidal against beta-lactamase-producing Escherichia coli; hence, the attachment of biologically active components to their surface expands their application in antimicrobial applications. This study has presented evidence of the antibacterial effects of $P$. grandiflora tuber extracts conjugated with gold nanoparticles and their synergistic capacity against selected pathogenic bacteria. A synergistic effect was observed against E. coli, K. pneumonia, and MSSA when gold nanoparticles conjugated to $P$. grandiflora tuber extracts. Hence, all conjugates with synergistic effects are claimed to be responsible for improving the effectiveness of many extracts and conventional antimicrobial drugs.

\section{Data Availability}

The data used to support the findings of this study are available from the corresponding author upon request.

\section{Conflicts of Interest}

The authors declare that they have no conflicts of interest.

\section{Acknowledgments}

The present study was funded by the South African National Research Foundation grant no. IRF18021531377 to Prof A. Samie and grant no. 107935 to Dr K. Pillay and the University of Venda Research and Publication Committee.

\section{References}

[1] R. Y. Pelgrift and A. J. Friedman, "Nanotechnology as a therapeutic tool to combat microbial resistance," Advanced Drug Delivery Reviews, vol. 65, no. 13-14, pp. 1803-1815, 2013.

[2] S. Iravani, "Green synthesis of metal nanoparticles using plants," Green Chemistry, vol. 13, no. 10, pp. 2638-2650, 2011.

[3] Y. Sun and Y. Xia, "Shape-controlled synthesis of gold and silver nanoparticles," Science, vol. 298, no. 5601, pp. 2176-2179, 2002.

[4] M. Singh, R. Kalaivani, S. Manikandan, N. Sangeetha, and A. K. Kumaraguru, "Facile green synthesis of variable metallic gold nanoparticle using padina gymnospora, a brown marine macroalga," Applied Nanoscience, vol. 3, no. 2, pp. 145-151, 2013.

[5] D. R. Bhumkar, H. M. Joshi, M. Sastry, and V. B. Pokharkar, "Chitosan reduced gold nanoparticles as novel carriers for 
transmucosal delivery of insulin," Pharmaceutical research, vol. 24, no. 8, pp. 1415-1426, 2007.

[6] V. Agostoni, P. Horcajada, M. Noiray et al., "A "green" strategy to construct non-covalent, stable and bioactive coatings on porous MOF nanoparticles," Scientific Reports, vol. 5, no. 1, pp. 7925-7934, 2015.

[7] R. Gannimani, M. Ramesh, S. Mtambo, K. Pillay, M. E. Soliman, and P. Govender, " $\gamma$-cyclodextrin capped silver nanoparticles for molecular recognition and enhancement of antibacterial activity of chloramphenicol," Journal of Inorganic Biochemistry, vol. 157, pp. 15-24, 2016.

[8] A. Murei, K. Pillay, P. Govender, N. Thovhogi, W. N. Gitari, and A. Samie, "Synthesis, characterization and in vitro antibacterial evaluation of pyrenacantha grandiflora conjugated silver nanoparticles," Nanomaterials, vol. 11, no. 6, p. 1568, 2021.

[9] L. F. Leopold, I. S. Tódor, Z. Diaconeasa et al., "Assessment of PEG and BSA-PEG gold nanoparticles cellular interaction," Colloids and Surfaces A: Physicochemical and Engineering Aspects, vol. 532, pp. 70-76, 2017.

[10] A. Samie, C. L. Obi, P. O. Bessong, and L. Namrita, "Activity profiles of fourteen selected medicinal plants from rural venda communities in South Africa against fifteen clinical bacterial species," African Journal of Biotechnology, vol. 4, no. 12, pp. 1443-1451, 2005.

[11] C. Singh, R. K. Baboota, P. K. Naik, and H. Singh, "Biocompatible synthesis of silver and gold nanoparticles using leaf extract of dalbergia sissoo," Advanced Materials Letters, vol. 3, no. 4, pp. 279-285, 2012.

[12] F. Cai, J. Li, J. Sun, and Y. Ji, "Biosynthesis of gold nanoparticles by biosorption using Magnetospirillum gryphiswaldense MSR-1," Chemical engineering journal, vol. 175, pp. 70-75, 2011.

[13] P. Singh, Y.-J. Kim, D. Zhang, and D.-C. Yang, "Biological synthesis of nanoparticles from plants and microorganisms," Trends in Biotechnology, vol. 34, no. 7, pp. 588-599, 2016.

[14] S. M. H. Abtahi, "Synthesis and characterization of metallic nanoparticles with photoactivated surface chemistries," Doctoral dissertation, Virginia Tech, Blacksburg, Virginia, 2013.

[15] P. Mukherjee, S. Senatipati, D. Mandal, A. Ahamad, and M. Kumar, "Extracellular synthesis of gold nanoparticles by fungus F. oxysporum," Nanotechnology Reviews, vol. 24, pp. 12-16, 2001.

[16] G. Southam and T. J. Beveridge, "The occurrence of sulfur and phosphorus within bacterially derived crystalline and pseudocrystalline octahedral gold formed in vitro," Geochimica et Cosmochimica Acta, vol. 60, no. 22, pp. 4369-4376, 1996.

[17] W. Jahnen-Dechent and U. Simon, "Function follows form: shape complementarity and nanoparticle toxicity," Nanomedicine, vol. 3, no. 5, pp. 601-603, 2008.

[18] W. Haiss, N. T. K. Thanh, J. Aveyard, and D. G. Fernig, "Determination of size and concentration of gold nanoparticles from UV-Vis spectra," Analytical Chemistry, vol. 79, no. 11, pp. 4215-4221, 2007.

[19] V. Amendola and M. Meneghetti, "Size evaluation of gold nanoparticles by UV-vis spectroscopy," The Journal of Physical Chemistry C, vol. 113, no. 11, pp. 4277-4285, 2009.

[20] G. Schmid, "The relevance of shape and size of Au55 clusters," Chemical Society Reviews, vol. 37, no. 9, pp. 1909-1930, 2008.

[21] M. F. Lengke, M. E. Fleet, and G. Southam, "Biosynthesis of silver nanoparticles by filamentous cyanobacteria from a silver (I) nitrate complex," Langmuir, vol. 23, no. 5, pp. 2694-2699, 2007.
[22] D. A. Kumar, V. Palanichamy, and S. M. Roopan, "Green synthesis of silver nanoparticles using alternanthera dentata leaf extract at room temperature and their antimicrobial activity," Spectrochimica Acta Part A: Molecular and Biomolecular Spectroscopy, vol. 127, pp. 168-171, 2014.

[23] M. Saravanan, S. K. Barik, D. MubarakAli, P. Prakash, and A. Pugazhendhi, "Synthesis of silver nanoparticles from bacillus brevis (NCIM 2533) and their antibacterial activity against pathogenic bacteria," Microbial Pathogenesis, vol. 116, pp. 221-226, 2018.

[24] S. Ahmed and S. Ikram, "Silver nanoparticles: one pot green synthesis using terminalia arjuna extract for biological application," Journal of Nanomedical Nanotechnology, vol. 6, no. 4, p. 309, 2015.

[25] S. Shrivastava, T. Bera, A. Roy, G. Singh, P. Ramachandrarao, and D. Dash, "Characterization of enhanced antibacterial effects of novel nanoparticles," Nanotechnology, vol. 18, pp. 103-112, 2007.

[26] D. Luseba, E. E. Elgorashi, D. T. Ntloedibe, and J. Van Staden, "Antibacterial, anti-inflammatory and mutagenic effects of some medicinal plants used in South Africa for the treatment of wounds and retained placenta in livestock," South African Journal of Botany, vol. 73, no. 3, pp. 378-383, 2007.

[27] A. A. Mostafa, A. A. Al-Askar, K. S. Almaary, T. M. Dawoud, E. N. Sholkamy, and M. M. Bakri, "Antimicrobial activity of some plant extracts against bacterial strains causing food poisoning diseases," Saudi Journal of Biological Sciences, vol. 25, no. 2, pp. 361-366, 2018.

[28] I. N. Vatsos and C. Rebours, "Seaweed extracts as antimicrobial agents in aquaculture," Journal of Applied Phycology, vol. 27, no. 5, pp. 2017-2035, 2015.

[29] A. Murei, W. B. Ayinde, M. W. Gitari, and A. Samie, "Functionalization and antimicrobial evaluation of ampicillin, penicillin and vancomycin with pyrenacantha grandiflora baill and silver nanoparticles," Scientific Reports, vol. 10, no. 1, pp. 1-14, 2020.

[30] A. Rosato, C. Vitali, N. De Laurentis, D. Armenise, and M. Antonietta Milillo, "Antibacterial effect of some essential oils administered alone or in combination with norfloxacin," Phytomedicine, vol. 14, no. 11, pp. 727-732, 2007. 